\title{
Android Controlled Smart Wheelchair with Gesture and Voice Control
}

\author{
M R Sreeraj ${ }^{1}$ Shahima Azad ${ }^{2}$ Binumol Baby ${ }^{3}$ Ms. Neema George ${ }^{4}$ \\ ${ }^{1}$ Dept. of computer science \& Engineering, India, msreeraj123@ gmail.com \\ ${ }^{2}$ Dept. of computer science\& Engineering, India, shahimaazad04@ gmail.com \\ ${ }^{3}$ Dept. of computer science\& Engineering, India, binumolbabay1998@gmail.com \\ ${ }^{4}$ Dept. of computer science \&Engineering, India, neema.george@mangalam.in
}

\begin{abstract}
Being disabled brings about a feeling of isolation from the outside world and a sense of dependability as we have to depend on others help for just moving from one place to other and many other basic needs. [1] Wheelchair has solved this problem to an extent as it's provides personal mobility for the aged and disabled. [1] [2] But it is very difficult for the disabled people to use the manual power of the wheelchair independently. As a solution to this problem, we have put forward a power wheelchair which can be controlled with a simple android device using IOT technology.[2] We have used the technology initially used to control robots to control a power wheelchair using an android device either by touch joysticks or by voice commands or by gesture.[1][5]
\end{abstract}

Key words: Android application, wheelchair, physicallychallenged, Arduino UNO, Internet of things,sensors, voice commands, Bluetooth device, DC motor, object detection.

\section{INTRODUCTION}

Physical disability is increasing due to aging, accidents and various diseases like paralysis. For this reason, the use of wheelchair is increasing. Some of the people who use the wheelchair can't operate the wheelchair with their hands. [8] [3]So, they need the help of others to move the wheelchair from one place to the other. The android controlled wheelchair can overcome these problems mostly by the idea of implementing the wheelchair using android application, now a day's android mobile phones are commonly used. [2]The wheelchair will be operated with the voice commands and gesture as inputs, which gives the directions as user directs. [3] This wheelchair has gained more independent existencebecause the Arduino helps to covert the voice commands into outputs and the wheelchair can move freely. Along with this an ultrasonic sensor is used to detect the obstacles. [9] By the proposed approach, the wheelchair will be working on the inputs such as voice, gesture and touch via an android phone that moves the wheelchair according to the commands provided. It. helps the physically disabled and aged people to move the wheelchair independently without any external support. [8]

\section{LITERATURE SURVEY}

In the past few years, many projects are developed related to the wheelchair. The existing wheelchair for the disabled and aged people are designed in such a way that they can't move independently without an external support. The developed projects are based on the joystick, voice, hand gesture and brain wave sensing. In the recent years, the battery powered wheelchairs also have been invented, which had gained popularity because it's more helpful for the physically disabled and the aged people.

[1] Thomas Roofer, Christian Mandel "Controlling an automated wheelchair via joystick, /Head joystick supported by smart driving assistance", here the smart driving assistance supports the user of the wheelchair in complex navigational situations.

[2] Mohammed Asger, Mirza Badra, Khan Irshad, Shaikh Aftab"Automated Innovative Wheelchair" on December 2013, focused on the sensors, radar and ultrasonic technologies which provide the electronic wheelchairs. 
M R Sreeraj et al ., International Journal of Advances in Computer Science and Technology, 9(6), June 2020, 34 - 38

[3] Kyung Mog Lee study on the electronic wheelchair controlled with a smart phone, here the author uses the android smart phone to control the wheelchair with voice commands.

[4] Sachin S. Patil, Kiran N. Patil, Sanjay P. Patil "Gesture based wheelchairs for physically disabled" focuses on the hand movements or the hand gesture to control the wheelchair.

[5] Holly A. Yanco, "Wellesley: A Robotic wheelchair system: Indoor navigation and user interface" focuses on the development and evaluation of a robotic intelligent wheelchair system based on the obstacle detection.

[6] Kohei Arai, Ronny Mardiyanto," Eyes based electric wheelchair control system" here the idea is to create an eye monitored system which helps the movements of wheelchair depending on the eye movements.

\section{SCOPE}

The existing wheelchairs have some limitations in their functions and flexibility. The android smart phones are become the part of our day to day life, because of that it can be effectively used as the medium to move the wheelchair independently. The wheelchair is controlled using the voice commands, gesture, and touch via the android phone. Using this user can control the wheelchair independently without any external support. The motivation behind this smart wheelchair using android platform is to build an automated wheelchair for the disabled and aged people to move from one place to another without seeking the help from any external individual.

\section{PROPOSED SYSTEM}

The purpose of this paper is to implement a smart wheelchair which moves independently as per the users input. The system is developed with the voice, phone gesture and touch as inputs via the android smart phone. The wheelchair is fully independent and the user does not need any external support to move the wheelchair. The wheelchair is implemented with incorporating the technologies of Arduino and motors. The Arduino is connected with the motors for the movement of wheelchair. Bluetooth module is incorporated in the wheel chair architecture and the android phone will be connected to the Bluetooth.

The inputs used are:

\subsection{VOICE}

When voice is selected as an input, the user can give the directions as voice commands for moving the wheelchair to the desired locations. The voice is taken from the android smart phone and is given to the Bluetooth module to control the operations of DC motor.

Common words and its operations:

\begin{tabular}{|l|l|}
\hline VOICE COMMANDS & OPERATIONS \\
\hline STOP & Stops moving \\
\hline FORWARD & Moves forward \\
\hline LEFT & Moves left \\
\hline RIGHT & Moves right \\
\hline BACK & Moves backward \\
\hline
\end{tabular}

\subsection{PHONE GESTURE}

On selection of gesture as input, the user will have to make the directions for the android device. Here the phone's sensors and accelerometers will be used to control the movements that the user gives.

\subsection{TOUCH}

If the input is taken as touch, the direction keys are front, back, left, right which decides the movements of the wheelchair. These inputs given through these keys will control the movement of the wheelchair to the desired locations.

\subsection{OBJECT DETECTION}

To ensure the safety of the disabled people, also the object detection sensors are used to detect the obstacles. This wheelchair prevents the users from accidents by providing the object detection phase. If any obstacles are detected it provides the instructions for the user's safety.

\section{IMPLEMENTATION}

Here we need both the software and hardware requirements in order for the implementation. 


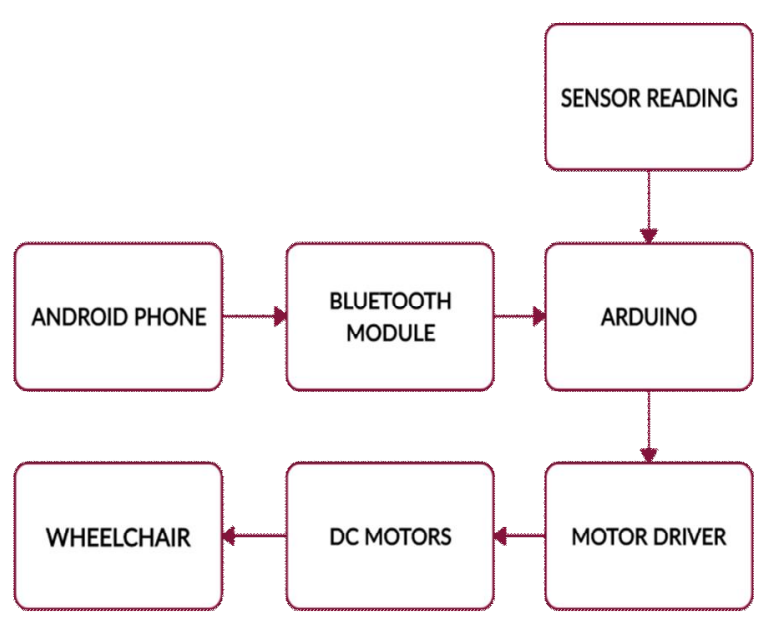

Figure 1: Wheelchair Block diagram

\subsection{SOFTWARE REQUREMENTS}

\section{Android Application}

Android software is used to control the wheelchair.

\section{Coding language}

A coding language such as $\mathrm{c}$ or python is required to program the Arduino.

Arduino Software (IDE)

It is used to create and upload the code to the Arduino.

\subsection{HARDWARE REQUREMENTS}

\section{Arduino UNO}

It is a Microcontroller board which here is used to control the wheelchair.

\section{Motor}

Two DC motors are used here for the movement of the wheelchair.

\section{Bluetooth module}

HC-05 Bluetooth module is used here for wheelchair and android mobile interface.

\section{Battery}

A suitable battery is used to power the wheelchair.

\section{Android phone}

An android phone with the wheelchair application is needed to control the wheelchair.

\section{Sensor}

HC-SR04 ultrasonic sensor is used for object detection.

\section{Wheelchair chassis}

A wheelchair chassis is needed to mount these components.

\subsection{INTERFACE DESIGN}

The figure given shows the system interface for the proposed application. The system is created for the android mobile phone would contain the three primary inputs that are voice, gesture and touch. [3] For the wheelchair processing, each of these modules transmit the data through the medium. These data are transmitted through the Bluetooth module for the transmission and receiving. The android application can be written using the $\mathrm{c}$ language. As per the users demand the dc motor is coupled with Arduino. [3]

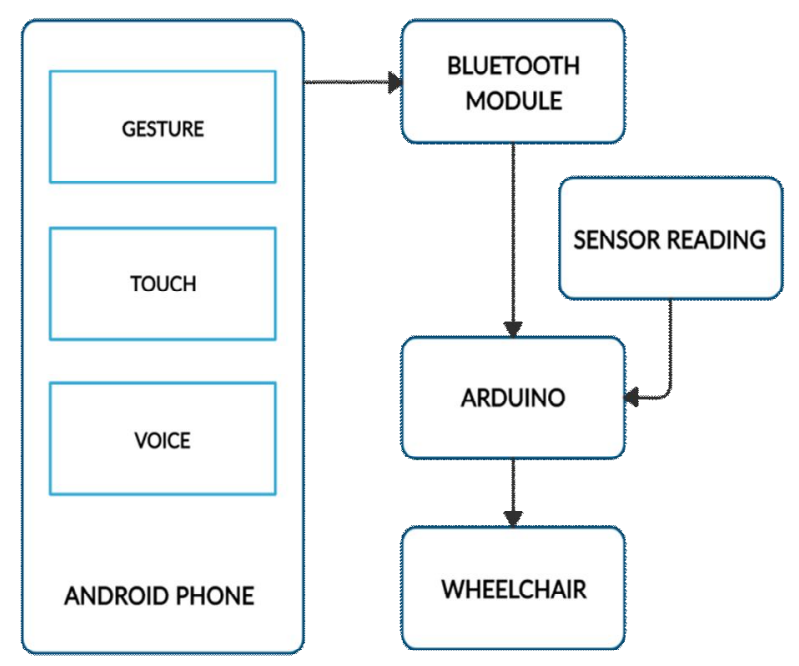

Figure 2: Wheelchair-Arduino Interface diagram

\section{ADVANTAGES}

User friendly: the method employed for the working of wheelchair is user friendly since it can be used in any of the three ways convenient for the user.

Reduce man power: the manual work is reduced to its minimum since touch, voice;gestures are there to control the wheelchair.

Less wiring because of Bluetooth: wiring could increase complexity because the aged and disabled would find it difficult to handle.

Automated operation: all the three methods employed could bring the whole device to a fully automated one. 


\section{RESULT AND ANALYSIS}

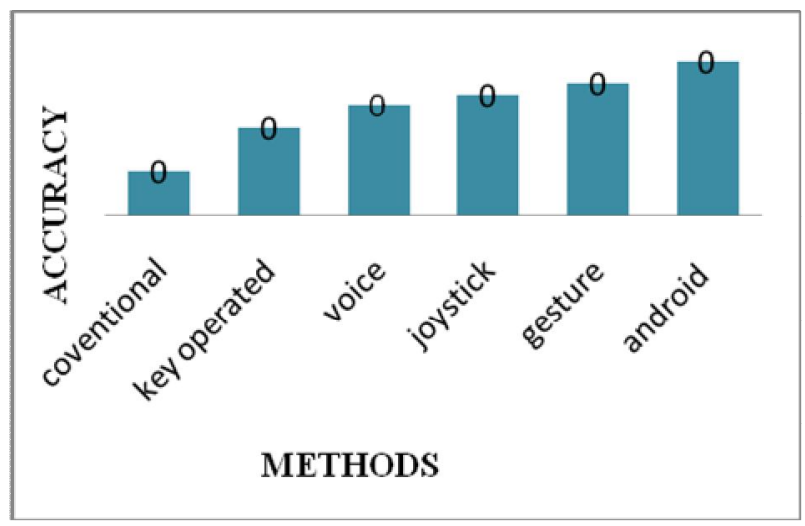

Here Android refers to our android controlled wheel chair whose efficiency and accuracy are greater than that of the all other specified papers. The specified model helps in bringing up a user-friendly technology for handling the user's needs in movement because any of the preferred ways of movement can be chosen according to the users convenience whereas all other meters of comparison here uses only a single technology and is not up to the fullest consent of the user.

\section{FUTURE SCOPE}

The next phase development that can be incorporated to this project is the addition of brain wave control which could be an ice breaker for the wheelchair industry. The brainwave-controlled wheelchair is connecting the brain to the computer for getting structure and information of the brain where the thoughts of movement will be taken as input signals and quicker movement will be possible. Electroencephalography is used for the connection between the computer and brain. This type of wheelchair is used for the paralyzed and handicap people. The EEG signals help the movement of the wheelchair to move freely. The system can store the video and the wheelchair can independently recognize the various patterns. The brain wave sensing could do much more with the aged and the disabled as they can be more conveniently operated by themselves.

\section{CONCLUSION}

The smart wheelchair consist of voice, gesture, touch and object detection that we intend to find a cost effective design to build a wheelchair for physically disabled people, who can independently move the wheelchair for their displacement. [3] The existing system needs the help of other people to move the wheelchair from one place to another. Our proposed system aims to resolve the above issue. This project provides the users to control the wheelchair automatically using an android phone because the user interface of an android smart phone is simple. This is an IOT based wheelchair which is controlled using an android smart phone taking inputs as phone gesture, voice, and touch.[3] Also it provides object detection that provides ease of navigation without colliding with objects in its path, so it assures the safety of the wheelchair users. We are trying to develop a system which could make the disabled people one independent and provide an extreme ease in their life. Project involves the hardware designing and software knowledge.

\section{REFERENCES}

[1] Thomas Rofer, Tim Laue and Christian Mandel: "Controlling an automated wheelchair via joystick/head joystick supported by smart driving assistance" IEEE, 2019.

[2] Richard C. Simpson, in the Journal of Rehabilitation Research and Development, "Smart wheelchairs: A Literature survey.

[3] Mohammed Asgar, MirzaBadra, Khan Irshad, Shaikh Aftab, "Automated innovative wheelchair" in December 2013, International Journal of Information Technology Convergence and Services.

[4] Holly A. Yanco, "Wheelesley: A robotic Wheelchair system: indoor navigation and user interface", MIT Artificial Intelligence Laboratory 545 Technology Square.

[5] Sachin S. Patil, Kiran N. Patil, Sanjay P. Patil: "Gesture based wheelchairs for physically disabled", it focus the hand movements and the hand gesture for controlling the wheelchairs. 
M R Sreeraj et al ., International Journal of Advances in Computer Science and Technology, 9(6), June 2020, 34 - 38

[6]Masato, Nishimori, Takeshi Saitoh and RyosukeKonishi, "Voice controlled intelligent wheelchair,"

[7]H.R. Singh, A Mobin, S Kumar, S Chauhan and S SAgarwal, "Design and Development of Voice/Joystick Operated Microcontroller Based Intelligent Motorized Wheelchair", Tencon 99. Proceedings ofthe IEEE Region 10 Conference, Vol 2, pp. 1573 - 1576, Feb-1999.

[8]Rahul CM, NaliniClyer Voice and Accelemeter controlled Wheelchair, IJERECE, and vol: 2 (11), Nov 2015

[9]Simpson RC. Smart wheelchairs: A literature review. J Rehabil Res Dev. 2005; 42(4):423-36.

[10]Ozdalga E, Ozdalga A, Ahuja N. The smartphone in medicine: a review of current and potential use among physicians and students. J Med Internet Res. 2012; 14(5):e128. Published 2012 Sep 27. doi:10.2196/jmir.1994. 